\title{
A Review on ICT Based Applications for Intervention and Assistance of People with Memory Deficits
}

\author{
http://dx.doi.org/10.3991/ijet.v8i5.3009
}

\author{
Athanasios Drigas, Athanassia Dourou \\ NCSR 'Demokritos', Athens, Greece
}

\begin{abstract}
- this is a review paper which examines the exploitation of Information and Communication Technologies (ICTs) for cognitive deficits and more precisely for memory deficits. Several research papers which related to computerized techniques and developed applications for short or long term memory were chosen. In addition it is assessed in several presented papers of how helpful these techniques and applications prove to be for people with such deficits. This kind of assistance and intervention focuses on humancomputer interaction and on its positive results in the development of linguistic or social skills and personal relationships. This review, points out certain technological breakthroughs of several researchers from the Global Scientific Society that assist adults and children with memory deficits.
\end{abstract}

Index Terms-memory, assistance, long-term, shortterm,deficits, Ict applications on memory, ICTs.

\section{INTRODUCTION}

The role of technology for people with cognitive difficulties is crucial in the areas of based applications and neural networks. Memory is a key component of cognition that plays an integral role in cognitive development. Memory can be broken down into a number of domains including long term and short term memory. Short term memory's main part is working memory; long term memory consists of a declarative, procedural and perceptual representation system. Memory impairments can have negative consequences on social factors and the sense of personal history. Additionally, this kind of deficits may affect academic performance, particularly arithmetic and can result in low self-esteem.

Computerized programs seem to be a promising new approach to memory interventions. A quite important and recently developed area is ambient intelligence living [1]. The general goal of assistive living is to enable people with specific demands, such as elderly people, by applying ambient intelligence technology. Different ambient assisted living solutions set higher demands on the system quality and generally on software or system engineering. Further research has been done on Assisted Living Laboratory that used to train elderly people to use designed interfaces e.g. in case of an emergency.

Special instructors (teacher, parents) can have the opportunity to adapt these programs on requested needs. Further examination of how the type of trainer - teacher, researcher, and parent- could influence the outcome is needed. A recent development of dynamic media (video, animation) as a type of tool for educational purposes demonstrates not only technological but cognitive aspects of this tool. A research $\lceil 2\rceil$ examines how this type of media supports students' learning better than static media (text or images). After a proper investigation, results revealed that learning performance using dynamic media was higher than those of static and the more complex the learning material was, the larger was the benefit using dynamic media.

Another promising tool $\lceil 3\rceil$ that was recently developed in order to optimize the procedure of learning by combining short time and high quality is Mnemo Wiki that filters, adapts and visualizes content inside a Wiki knowledge base. It appears to be a quite useful tool and with further research and development it could assist e- learning process using web 2.0 technologies or might be used as a tool for studying mental models.

Conclusively, the studies examined have significant impact on special or general areas of technology (artificial intelligence etc.) and on the area of psychological and personal assessment. They focus on the assistance of people with memory deficits which were caused by heredity factors, accidents, learning disorders or aging. However, more research is needed to determine the factors that lead to the best outcomes in computerized memory interventions.

\section{SHORT TERM-MEMORY}

\section{A. Working Memory}

Certain tools for memory training have been developed; one of them is Cogmed [4]. This program has been marketed at schools to assist students' performance and was available as clinical practice for treatment of ADHD. There are three types of Cogmed training for preschoolers and older children. It includes several visuo-spatial and verbal memory tasks that have been embedded in videogames. Although initial studies indicated that Cogmed might improve reasoning abilities, after several studies it was proved that replications had failed. Moreover, it is suggested to improve attention as it relates to selecting appropriate information or controlling impulses. Finally, it was noted that working memory capacity and performance may improve, although more research is needed to be done.

Other studies focused on working memory's limit capacity (the amount of information that can be held) [5]. Two user studies that asked users to perform certain data analysis tasks were realized. The first one used transfer function for comparison and had high demands from the 
users. During this test it was found that limited working memory capacity has impact on the effectiveness of transfer function. The second one was related to virtual environments, where the subject was assimilated as it should memorize the data structure in order to perform the task. Finally it was found that cognitive abilities and working memory capacity are really determining factors for the success or failure of a 3D interface.

Another study [6] presented several well known neuropsychological tests of working memory performance whose results are shown through using a computer program which can be found on line or runs in a Dos window on a pc. Tasks, such as Corsi blocks test, the multiple location search task and the letter recitation task, include the subject-ordered pointing task. For better statistical analysis the computer program used examples extracted from the statistical procedures above for single case and group case analysis.

\section{LONG-TERM MEMORY}

This kind of memory may consist of the following subparts: declarative and procedural [12] . Declarative memory is divided into two types: episodic and semantic memory. Episodic or autobiographic memory stores information about events-episodes while semantic memory refers to inferable knowledge and word knowledge. Procedural memory on the other hand is relative to motor skills. Each of them has been used for computerized accompaniments in order to train certain agents or develop additional software to train memory or improve certain deficits.

Certain type of cognitive impairment is more frequent in the elderly. There was a study on developing a cognitive orthotic system called Autoreminder [8] which was intended to help older people adapt to this type of cognitive deficit and make their everyday life easier by using a range of AI techniques. Autoreminder serves as a cognitive orthotic, providing its users-clients with reminders for their daily initial activities. This system was written in Java and Lisp for a Wintel platform and it has been tested in the laboratory. Its current version is deployed on a mobile robot, named "Pearl", and it is being developed as a part of the Initiative on Personal Robotic assistance for the elderly (the Nursebot project). People were quite satisfied by using this system and found it useful. More extensions and modules are planned to be added and result into more facilities.

A single case study has been done in order to illustrate the potential related to neuropsychological deficits that computer programs can train [9]. More precisely the computer training of an adult with severe head injury and considerable problems with memory has been done. Several sessions were carried out over a six- week period and a computer program was used (Johnson, 1989). This program gave the opportunity to experiment with strategies for learning word lists, and measure the results of his training under different conditions. Using the computer aided motivation and may be used as a therapeutic tool especially when teaching coping strategies. Finally, computers may provide personalized assessment and independence when a person is training.

It is a fact that many people suffer from different types of memory impairments at a point in their life. A system was designed for people with such deficits, called "Neu-
roPage" [10]. Most conventional memory aids expect lots of memory usage, which is difficult and sometimes impossible for the user to do. NeuroPage is a computer based reminder system, which keeps complexity of operation "behind the scenes" and users keep a simple device. In order to achieve this collaboration a combination of computing and telecommunication was used. Its three main components are pager, computer and paging company. Although,there were certain usability problems, results have shown that NeuroPage is an effective reminding device in cases of severe memory impairment. It gives the opportunity for further investigation and the development of other components.

\section{A. Episodic Memory}

An implementation of episodic memory model for artificial companions was created [11]. The information stored in our episodic memory reflects past events and a certain data structure for the corresponding event data has been elaborated. For the process of knowledge within the memory model data mining techniques were used. In order to classify results of this data mining task, two Bayesian classifiers were applied. This is the first approach in an effort to incorporate data mining techniques to episodic memory modeling for artificial companions; further research is needed to be done to facilitate this kind of creations even more.

\section{B. Semantic memory}

A research has been undertaken, concerning semantic memory, which stores a person's general knowledge of the world. Certain studies have been made on the field of psychology and were implemented in cognitive architecture, for example ACT - R [13]. This type of memory has not yet been found on functionally-based cognitive architectures such as Soar's. During this research the main purpose was to study the general computational functionalities of semantic memory, its interactions with cognitive architecture and solving several tasks concerning learning, which means that researchers tried to develop a new component into Soars. Here the motivations, the implementations and the design are presented but there is further research to be done in order to get the results of a simple task.

\section{Procedural memory}

Concerning this type of memory certain studies were held on how people with memory deficits could acquire complex knowledge and the processes required to perform a computer entry task [7]. Previous studies mostly focused on people with amnesia but this study is demonstrated on people with brain damage, memory disorders of variable severity and other cognitive deficits. All patients were able to learn data entry procedures and information related to the task. Although declarative knowledge was found at slower rates during tasks, procedural was at the same level in patients and other control subjects. Finally transformation of declarative knowledge was observed during procedural tasks.

Another research developed several ways to facilitate learning through video and animations [14]. The study investigated the impact of presenting together a video recording and a series of static pictures. There were held out two experiments and resulted that dynamic presentation of pictures was more effective to static pictures mode and 


\section{SHORT PAPER}

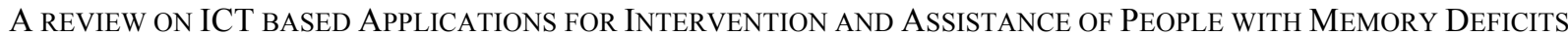

showing fewer pictures was more beneficial. Finally there was found effectiveness of a combination of instructional animation with static pictures, where the number of pictures that was used was an important factor.

\section{CONCLUSION}

During this review paper an attempt was made to present the significant role that ICTs play in dealing with memory deficits. The most important parameters about memory training are the way traditional techniques were adapted and computerized in the field of ICTs on the one hand and the design and development of completely new tools and techniques on the other. By using these applications and following these techniques, people with various cognitive difficulties may take part in the learning experience and improve their everyday life.

\section{REFERENCES}

[1] A. Holzinger, M. Kickmeier-Rust, and D. Albert, "Dynamic Media in Computer Science Education; Content Complexity and Learning Performance: Is Less More?" Journal of Educational Technology \& Society, vol. 1, issue 11, pp. 279-290, (2008).

[2] C. Stickel, M. Ebner, A. Holzinger, "Useful Oblivion Versus Information Overload in E-Learning Examples in the Context of Wiki Systems,"Journal of Computing and Information Technology, vol. 16, issue 4, pp. $271-277,2008$.

[3] T. Kleinberger, M. Becker, E. Ras, A. Holzinger, P. Müller, "Ambient Intelligence in Assisted Living: Enable Elderly People to Handle Future Interfaces". LNCS 4555.

[4] Z. Shipstead, K. Hicks, R. Engle, "Cogmed working memory training: Does the evidence support the claims?" Journal of Applied Research in Memory and Cognition, vol. 1, issue 3, pp. 185193, September 2012. http://dx.doi.org/10.1016/ j.jarmac.2012.06.003

[5] R. Gillett, "Assessment of Working Memory Performance in SelfOrdered Selection Tests Cortex", vol. 43, issue 8, pp. 1047-1056, 2007.

[6] W. Qi, "How Much Information Do You Remember? -The Effects of Short-Term Memory on Scientific Visualization Tasks," Virtual Reality, Lecture Notes in Computer Science, vol. 4563, pp 338347, 2007.

[7] E. L. Glisky, "Acquisition and transfer of declarative and procedural knowledge by memory-impaired patients: A computer data- entry task," Neuropsychologia, vol. 30, pp. 899-910, 1992. http://dx.doi.org/10.1016/0028-3932(92)90034-J

[8] M. Pollack, L. Brown, D. Colbry, C. McCarthy, C. Orosz, B. Peinter, S. Ramakrishnan, I. Tsamardinos, "Autominder : an Intelligent cognitive orthotic system for people with memory impairment," Robotics and Autonomous Systems, vol. 44, issue 3-4, pp. 273- 282, 2003 http://dx.doi.org/10.1016/S09218890(03)00077-0

[9] C. D. Nugent, "ICT in the elderly and dementia, Aging \& Mental Health," vol.11, issue 5, pp. 473-476, 2007. http://dx.doi.org/10.1080/13607860701643071

[10] E. Svoboda, B. Richards, L. Leach, V. Martens, " PDA and smart phones use by individuals with moderate to severe memory impairment: Application of a theory - driven training programme," Neuropsychological Rehabilitation : An International Journal, vol. 21, issue 6, 2011.

[11] M. U Keysermann, A.A. Freitas, and P.A. Vargas, "Implementing a data mining approach to episodic memory modelling for artificial companions," Proceedings of AISB'11: Human Memory for Artificial Agents, Society for the Study of Artificial Intelligence and the Simulation of Behaviour, pp. 41-48, 2011.

[12] A. Baddeley, " The episodic buffer: a new component of working memory?" Trends in cognitive Science, vol. 11, issue 4, pp. 417423, 2000. http://dx.doi.org/10.1016/S1364-6613(00)01538-2

[13] Y. Wang, J.E Laird, "Integrating semantic memory into a cognitive architecture," Technical Report CCA-TR-2006-02, University of Michigan, 2006.

[14] A. Arguel, E. Jamet, "Using video and static pictures to improve learning of procedural contents," Computers in human behavior vol. 25, pp. 354-359, 2009.

\section{AUTHORS}

A. Drigas is with N.C.S.R. 'Demokritos', Institute of Informatics and Telecommunications, Telecoms Lab - Net Media Lab, Agia Paraskevi, 153 10, Athens, Greece(email : dr@iit.demokritos.gr)

A. Dourou is with N.C.S.R. 'Demokritos', Institute of Informatics and Telecommunications, Telecoms Lab - Net Media Lab, Agia Paraskevi, 153 10, Athens, Greece (athdou@gmail.com)

Submitted 15 July 2013. Published as re-submitted by the authors 13 October 2013. 